\title{
Editorial: Intelligent Concrete, New Functionalities and Nanotechnology
}

\author{
Wengui $\mathrm{Li}^{1 *}$, Maria Konsta-Gdoutos ${ }^{2}$, Xianming Shi ${ }^{3}$, Konstantin Sobolev $^{4}$ and \\ Surendra P. Shah ${ }^{2}$ \\ ${ }^{1}$ School of Civil and Environmental Engineering, University of Technology Sydney, Sydney, NSW, Australia, ${ }^{2}$ Center for Advanced \\ Construction Materials, The University of Texas at Arlington, Arlington, TX, United States, ${ }^{3}$ Department of Civil and Environmental \\ Engineering, Washington State University, Pullman, WA, United States, ${ }^{4}$ Department of Civil and Environmental Engineering, \\ University of Wisconsin-Milwaukee, Milwaukee, WI, United States
}

Keywords: intelligent concrete, self-sensing, self-healing, durability, photocatalysis, nanotechnology

\section{Editorial on the Research Topic}

Intelligent Concrete, New Functionalities and Nanotechnology

Cement-based concrete is the most used construction material in the world, ubiquitous in most of the infrastructures. Concrete infrastructure, however, is at the risk of premature failure when the degradation of concrete compromises their serviceability and reliability. The risk stems from complex interactions between concrete and the service environment, absence of advanced design and condition assessment approaches, and timely preservation or maintenance. Significant efforts are needed to maintain the aging infrastructure at a serviceable and safe state. Recent advances in nanotechnology and bio-concrete hold great promise to address these issues and potentially adding new functionalities and yet unexplored areas of self-sensing and self-healing properties. Examples of intelligent materials include concrete capable of sensing the variations of environmental and loading conditions and adopt suitable responses by altering one or more working parameters during their service life.

In comparison to conventional concrete, intelligent concretes can be designed to optimize their strengths, serviceability, durability, and other functions of infrastructure thereby reducing life-cycle costs, consumption of resources and associated environment impacts. All these functional features help lay a solid material foundation for building smart cities and sustainable built environments. Intelligent concrete is essential to the implementation of durable and smart infrastructures that feature properties and functionalities notably different from conventional concrete, such as selfsensing, self-healing, self-cleaning, featuring energy-harvesting abilities and storage, electromagnetic-shielding, photocatalytic, and hydrophobic properties. Intelligent concrete also contributes to the resilience of infrastructures, with its ability to react upon an external stimulus, such as stress, deformation, humidity, and temperature.

These published research studies on this research topic aim to cover original or review articles exploring the innovation in intelligent concrete. Themes of seven studies include 1) Piezoresistivity of concrete containing steel fibers, carbon black, and graphene; 2) Impact properties of recycled aggregate concrete with nanosilica solution and polyvinyl alcohol fiber; 3) Zeolite-loaded titanium dioxide photocatalytic cement-based materials for efficient degradation of drinking water disinfection byproduct trichloroacetamide; 4) Aggressive environment resistance of concrete modified with nano alumina and nano silica; 5) Mechanism of microcapsule sustained-releasetype hydration heat inhibitor on low-heat portland cement; 6) Self-sensing capability of ultra-high performance concrete; and 7) Resistivity signature of graphene-based fiber-reinforced composite subjected to mechanical loading.

Liu et al. incorporated different scales of conductive materials to cement-based composites, including macro-scale steel fibers, micro-scale carbon black powder, and nano-scale graphene to 
ensure the improved strength, lower cost, and comparable pressure-sensitive performance by the coupling effect of three scales of materials. They found that the strength of intelligent concrete with multi-scale conductive materials is higher than that of the contrast group of ordinary concrete and intelligent concrete when adding nano-scale graphene alone. Especially, the addition of steel fibers significantly improved the crack resistance of the intelligent concrete. Wang et al. studies the dynamic impact properties of the modified recycled aggregate concrete (RAC) mixed with $2 \%$ nanosilica solution and polyvinyl alcohol (PVA) fibers using split Hopkinson pressure bar (SHPB). They reported that the stress and dynamic peak strain value of the specimen increased with the increase of the strain rate; moreover, with the increase of the RA replacement percentage and the PVA fiber content, the dynamic peak stress of the specimen decreases, while the dynamic peak strain, and the DIF value increase. She et al. used a two-step method to load $\mathrm{TiO}_{2}$ on a cement matrix, and zeolite as intermediate support, and then prepared $\mathrm{TiO}_{2} @$ Zeolite composite coated photocatalytic cementbased material (PCM). They studied the photocatalytic degradation of trichloroacetamide (TCAcAm) to evaluate its photocatalytic efficiency, and found that $\mathrm{TiO}_{2} @ Z$ Zeolite composite can improve the adsorption ability of $\mathrm{PCM}$ as $\mathrm{TiO}_{2}$ particles were dispersed on the surface of PCM homogeneously providing abundant active sites for photocatalytic reactions. The synergetic effect of $\mathrm{TiO}_{2} @$ Zeolite composite can remarkably improve the photocatalytic degradation rate, reaching up to 97.8\%. Xie et al. investigated the impacts of $\mathrm{CaCl}_{2}$ along with freeze/thaw and wet/dry cycles on the nano $\mathrm{SiO}_{2}$ and $\mathrm{Al}_{2} \mathrm{O}_{3}$ modified concrete. They reported that the addition of nanoparticles has a distinctive effect on the environment resistance enhancement of concrete samples. The microstructure analysis demonstrates that with the addition of nanoparticles, highdensity hydration products were formed, and which is beneficial to the properties enhancement of concrete products. Jia et al. prepared a kind of microcapsule sustainedrelease-type hydration heat inhibitor (MSR). They reported that the MSR had good regulating effect on hydration of low-heat Portland cement. When the dosage of MSR was $0.3 \%$, the rate of heat release was decreased by $10 \%$, and the peak temperature was decreased by $52 \%$. The 3 -days compressive strength decreased by $50 \%$, and the 28-days strength was the same as control. Wang et al. reviewed the recent advances in self-sensing capability of various Ultra-high performance concrete (UHPC)-based materials with the focus on sensing capability and mechanisms. They concluded that with the incorporation of micro steel fibers, carbon nanotubes, carbon nanofibrils, or nanographite platelets, it opens up great potential to allow UHPC to effectively sense stress, strain, and crack damage. Therefore, the UHPC-based structures can achieve the functionality of structure health monitoring (SHM). Sobolev et al. used a small quantity $(0.25 \%$ by weight of cement) of nano-sized conductive particles of graphene to change conventional portland cement-based composite into a semiconductive stress-sensing material. They investigated the piezoresistivity response of fiber-reinforced cement mortar with graphene nano-platelets, and reported that the electrical resistivity of the graphene-cement composite material can be used for structural health monitoring and damage detection.

Despite of the increasing amount of research published thus far on intelligent concrete, many challenges and research barriers remain unresolved calling for further innovative exploration. This research topic will focus on the new advances in theories, concepts, mechanisms, models, and practices related to new functionalities, advancing the field of smart concretes and structures, and addressing the challenges in the development and applications of intelligent infrastructure. The Guest Editorial team is confident that the successful application of intelligent and functional concrete using nanotechnology will facilitate the fundamental research and technology development of smart concrete and infrastructure. We would like to thank all contributing authors to the research topic, and the editorial staff of Frontiers in Materials for making this research topic possible.

\section{AUTHOR CONTRIBUTIONS}

All authors listed have made a substantial, direct, and intellectual contribution to the work, and approved it for publication.

\section{FUNDING}

The authors appreciate the Australian Research Council (DE150101751, DP220101051) and the US National Science Foundation (NSF).

Conflict of Interest: The authors declare that the research was conducted in the absence of any commercial or financial relationships that could be construed as a potential conflict of interest.

Publisher's Note: All claims expressed in this article are solely those of the authors and do not necessarily represent those of their affiliated organizations, or those of the publisher, the editors and the reviewers. Any product that may be evaluated in this article, or claim that may be made by its manufacturer, is not guaranteed or endorsed by the publisher.

Copyright $\odot 2022$ Li, Konsta-Gdoutos, Shi, Sobolev and Shah. This is an open-access article distributed under the terms of the Creative Commons Attribution License (CC $B Y)$. The use, distribution or reproduction in other forums is permitted, provided the original author(s) and the copyright owner(s) are credited and that the original publication in this journal is cited, in accordance with accepted academic practice. No use, distribution or reproduction is permitted which does not comply with these terms. 